\title{
Enhancement of the Adaptive Shape Variants Average Values by Using Eight Movement Directions for Multi- Features Detection of Facial Sketch
}

\author{
Arif Muntasa ${ }^{1}$, Mochammad Kautsar Shopan ${ }^{1}$, Mauridhi Hery Purnomo $^{2}$ \& \\ Kondo Kunio ${ }^{3}$ \\ ${ }^{1}$ Informatics Engineering Department, Trunojoyo University, \\ Kamal 16912, East Java, Indonesia \\ ${ }^{2}$ Electrical Eng. Department, Institut Teknologi Sepuluh November, \\ Surabaya 60111, Indonesia \\ ${ }^{3}$ MTC Laboratory, Tokyo University Technology, Katakuramachi, Hachioji-shi, \\ Tokyo 192-0982, Japan \\ Email: arifmuntasa@trunojoyo.ac.id
}

\begin{abstract}
This paper aims to detect multi features of a facial sketch by using a novel approach. The detection of multi features of facial sketch has been conducted by several researchers, but they mainly considered frontal face sketches as object samples. In fact, the detection of multi features of facial sketch with certain angle is very important to assist police for describing the criminal's face, when criminal's face only appears on certain angle. Integration of the maximum line gradient value enhancement and the level set methods was implemented to detect facial features sketches with tilt angle to 15 degrees. However, these methods tend to move towards non features when there are a lot of graffiti around the shape. To overcome this weakness, the author proposes a novel approach to move the shape by adding a parameter to control the movement based on enhancement of the adaptive shape variants average values with 8 movement directions. The experimental results show that the proposed method can improve the detection accuracy up to $92.74 \%$.
\end{abstract}

Keywords: control of the shape movement; enhancement of the adaptive shape variants average values; eight movement directions; facial sketch multi features.

\section{$1 \quad$ Introduction}

Biometrics is one part of the digital image processing used to identify a person through physical and behavioral characteristics. Currently, researches related to biometrics, such as face recognition [1]-[5], facial sketch features detection [6],[7], face tracking [8], face alignment and analysis [9], face sketch synthesis and recognition [10]-[13], and facial features detection [14]-[17] are gaining significant attentions. Facial features are one of deformable object detection as the face has thousands model when its expression is changed. The result of

Received June $16^{\text {th }} 2011$, Revised October $18^{\text {th }} 2011$, Accepted for publication October $29^{\text {th }}, 2011$.

Copyright $(92012$ Published by LPPM ITB, ISSN: 1978-3086, DOI: 10.5614/itbj.ict.2012.6.1.1 
facial features detection is influenced by several factors such as facial pose, distance between camera and face, exposure, facial expression, difference of image modality between the training and the testing set.

Multiple facial sketch detection has been conducted by using the different modality, photograph as the training set and face sketch as testing set. "The maximum line gradient method" has been proven capable to detect multi feature of the hatching face sketch, but this method has limitation as shapes always move to the maximum line gradient. If the maximum line gradient value feature is less around the hatch, the shapes will move away of the corresponding feature. To improve this method "the pyramid parameter model method" has been proposed [6], the experimental results show that multi feature detection of face sketch obtained up to $93.3 \%$ of accuracy. However, this method has also a weakness, the shape cannot move to the corresponding features when it lies on the same gradation. Both "The maximum line gradient method" and "the pyramid parameter model method" use frontal view face sketch as testing set. If the hatching face sketch with tilt up to about 15 degrees is tested, then the result detection obtained less than $80 \%$. On further development, "integrating the maximum line gradient value improvement and the level Set method variation" has been tested by using the hatching face sketch with tilt up to about 15 degrees and experimental results show that detection accuracy obtained is $89 \%$ [7]. The weakness of this method is caused by the presence of hatches around the feature that are considered as new objects so corresponding feature of the shape cannot be found. To overcome this limitation, we propose a new approach to improve the shape movement through the addition of the adaptive shape variants average value by using eight directions. Principally, our proposed method makes improvement simultaneous landmark movement based on previous movement. Improvement of landmarks is conducted by considering eight directions.

\section{Proposed Method}

In this research, 8 stages will be used to detect multi features of face sketch i.e. adjustment of translation and rotation, improvement of landmark initialization, dimensionality reduction by using principal component analysis, creating image gradation, improvement of the maximum line gradient method, the shape movement, integrating the level set method variation and improvement of maximum line gradient method to detect multi features (the first movement), and the adaptive shape variants average values with eight directions model to control shape movement (second movement), as seen in Figure 1. Training set used in this research is face images, whereas testing set used is hatching face sketches. For both training and testing set, images used are frontal with tolerance for limited side movement and limited tilt up to about 15 degrees. 


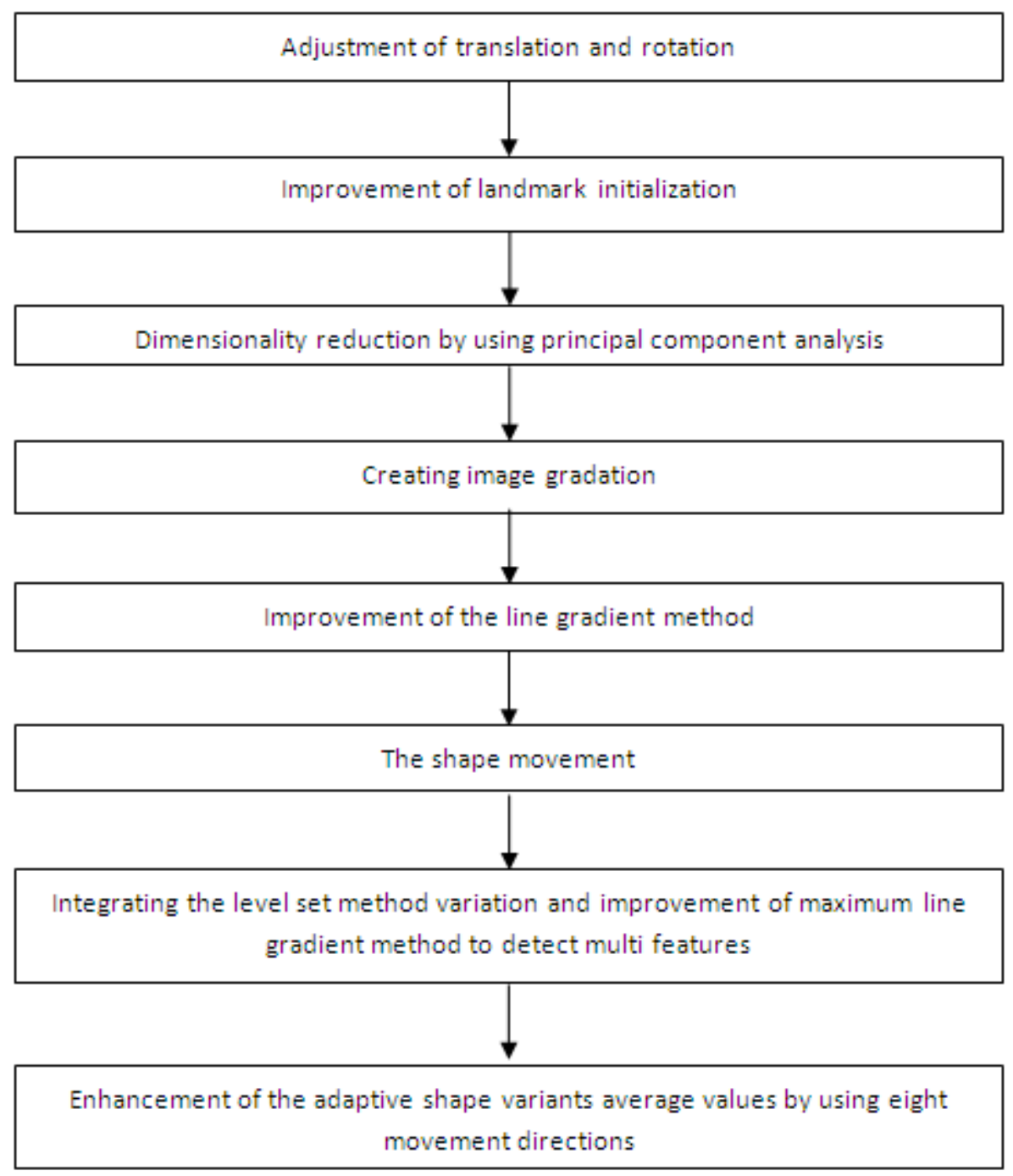

Figure 1 Framework system of our proposed method.

\subsection{Adjustment of Translation and Rotation [7]}

If $m$ represents number of training set, training set is represented by using $f(x, y)$, number of landmarks is symbolized by using $n$, index training set is represented by using $j$, whereas $i$ represents landmark index, then landmarks position of the training set can be expressed by using the following equation: 


$$
\left[\begin{array}{c}
X Y T_{1} \\
X Y T_{2} \\
X Y T_{3} \\
\ldots \ldots . . \\
X Y T_{m}
\end{array}\right]=\left[\begin{array}{ccccc}
\left(x t_{1,1}, y t_{1,1}\right) & \left(x t_{1,2}, y t_{1,2}\right) & \left(x t_{1,3}, y t_{1,3}\right) & \ldots & \left(x t_{1, n}, y t_{1, n}\right) \\
\left(x t_{2,1}, y t_{2,1}\right) & \left(x t_{2,2}, y t_{2,2}\right) & \left(x t_{2,3}, y t_{2,3}\right) & & \left(x t_{2, n}, y t_{2, n}\right) \\
\left(x t_{3,1}, y t_{3,1}\right) & \left(x t_{3,2}, y t_{3,2}\right) & \left(x t_{3,3}, y t_{3,3}\right) & & \left(x t_{3, n}, y t_{3, n}\right) \\
\ldots \ldots \ldots \ldots . . . & & & \ldots \ldots \ldots \ldots \ldots \\
\left(x t_{m, 1}, y t_{m, 1}\right) & \left(x t_{m, 2}, y t_{m, 2}\right) & \left(x t_{m, 3}, y t_{m, 3}\right) & \ldots & \left(x t_{m, n}, y t_{m, n}\right)
\end{array}\right]
$$

Principally, Eq. (1) will be used to obtain landmark initialization by adjustment of translation and rotation [7]. It is important to locate a shape close to the corresponding features. The closer the distance between the shape and the corresponding features, the higher accuracy can be achieved. The translation and rotation adjustment are sequence process used to obtain new landmark of training set. This process is begun by counting landmark average values for each training set and followed by calculating zero mean. The translation and rotation adjustment can be expressed by using the following equation:

$$
\phi_{j, i}=\phi_{j, i}+\overline{\phi m}_{j}
$$

where $\overline{\phi m}_{j}=\frac{\sum_{j=1}^{n / 2} \phi_{j, i}}{n / 2}$ and $\phi_{j, i}=\tan \left(\frac{x t_{j, i}}{y t_{j, i}}\right)^{-1}$

To achieve the middle point, it is necessary to calculate the value of $x t p_{j, I}$ and $x t p_{j, I}$ by using the following equation:

$$
\begin{aligned}
& x t p_{j, i}=r_{j, i} * \cos \left(\phi_{j, i}\right) \\
& y t p_{j, i}=r_{j, i} * \sin \left(\phi_{j, i}\right)
\end{aligned}
$$

In this case $r_{j, i}=\sqrt{\left(x t_{i}\right)^{2}+\left(y t_{i}\right)^{2}}, x t_{j, i}=x t_{j, i}-\overline{x t}_{j}$ and $y t_{j, i}=y t_{j, i}-\overline{y t}{ }_{j}$

\subsection{Improvement of Landmark Initialization [7]}

A landmark initialization position can be determined by using the following equation:

$$
\begin{aligned}
& X L I_{i}=\overline{X P}+\overline{\sigma x}_{i}+\eta \cdot \Delta \delta x_{i} \\
& Y L I_{i}=\overline{Y P}+\overline{\sigma y}_{i}+\eta \cdot \Delta \delta y_{i}
\end{aligned}
$$

In this case, $\eta$ represents adjustment constant value of the shape initialization. $\overline{X P}$ and $\overline{Y P}$ represent landmark average for $x t p$ and $y t p$ respectively, whereas 
$x t p$ and $y t p$ are the middle point of Eq. (3) and (4). $\overline{\sigma x}_{i}$ and $\overline{\sigma y}_{i}$ represent landmark variation average values of Eq. (1). Symbols of $\Delta \delta x_{i}$ and $\Delta \delta y_{i}$ represent zero mean middle point [7].

\subsection{Dimensionality Reduction by Using the Principal Component Analysis Method}

To reduce dimension of the training set features, it is necessary to compose new matrix as combining $x t p$ and $y t p$ values by using the following equation:

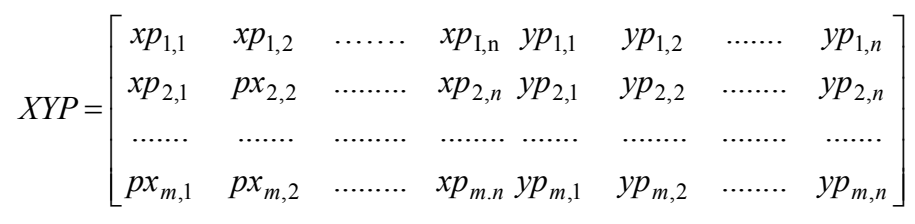

To simplify the calculation, Eq. (7) can be re-written in the following equation:

$$
X Y P=\left[\begin{array}{cccccccc}
x y p_{1,1} & x y p_{1,2} & \ldots \ldots . & x y p_{I, n} & x y p_{1, n+1} & x y p_{1, n+2} & \ldots \ldots . & y p_{1,2 n} \\
x y p_{2,1} & x y p_{2,2} & \ldots \ldots \ldots & x y p_{2, n} & y p_{2, n+1} & y p_{2, n+2} & \ldots \ldots \ldots & y p_{2,2 n} \\
\ldots \ldots . & \ldots \ldots . & \ldots \ldots \ldots & \ldots \ldots \ldots & \ldots \ldots . & \ldots \ldots \ldots & \ldots \ldots \ldots & \ldots \ldots . . \\
x y p_{m, 1} & x y p_{m, 2} & \ldots \ldots \ldots & x y p_{m . n} & y p_{m, n+1} & y p_{m, n+2} & \ldots \ldots \ldots & y p_{m, 2 n}
\end{array}\right]
$$

If $2 n=s$, then Eq. (8) can be changed in the following equation:

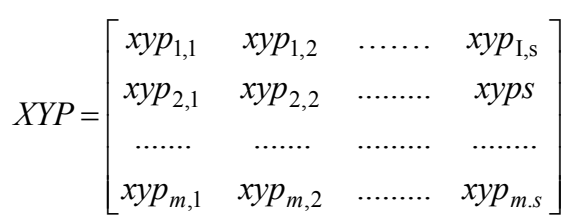

In this case $\mathrm{s}>>m$ and $s$ represents landmark dimension, $m$ represents number of training set. Based on Eq. (9), landmark average for all images can be computed by using the following equation:

$$
\mu_{i}=\frac{1}{m} \sum_{j=1}^{m} x y p_{j, i}
$$

Covariance value of can be derived from Eq. (9) and (10). It can be calculated by using the following equation:

$$
c=\frac{1}{m}\left(x y p_{j, i}-\mu_{j, i}\right)\left(x y p_{j, i}-\mu_{j, i}\right)^{T}
$$


To obtained eigenvalue and eigenvector, they can be computed by using the following equation:

$$
\begin{aligned}
& c \Lambda=\lambda I \Lambda \\
& (\lambda I-c) \Lambda=0 \\
& \operatorname{Det}(\lambda I-c)=0
\end{aligned}
$$

The results of Eq. (12) represents eigenvector $(\Lambda)$ and eigenvalue $(\lambda)$. Eigenvalue of $\lambda$ sorted in descending order and the corresponding eigenvector value of $\Lambda$ adjust to the eigenvalue of $\lambda$.

\subsection{Creating Image Gradation}

To simultaneously move shape toward the corresponding features, it is necessary to create image gradation. It can be conducted by using the first derivative followed by calculating the moving average value pixel surrounding neighbor. The most important thing is how many iterations the moving average value pixel with its neighbors is conducted. If number of iterations is performed too large, then the shape will move to incorrect area. Otherwise, the shape cannot move toward the corresponding features. Figure 2 show the result of image gradation using different iteration from the first row (left to right) and the second row (left to right), i.e. 2, 2, 3, 4, 5, 6 and 7 iterations respectively. If greater number of iterations is used, a greater gradient is obtained. To obtain an image gradation, the Sobel operator is used. It contains the horizontal and vertical gradient as seen in the following equation:

$$
G_{x}=\left[\begin{array}{lll}
-1 & 0 & 1 \\
-2 & 0 & 2 \\
-1 & 0 & 2
\end{array}\right] \text { and } G_{y}=\left[\begin{array}{ccc}
1 & 2 & 1 \\
0 & 0 & 0 \\
-1 & -2 & -1
\end{array}\right]
$$

The results of creating image gradient using Eq. (13) will be utilized to calculate the magnitude image as seen in the following equation:

$$
G[f(x, y)]=\sqrt{\left(G_{x} f(x, y)\right)^{2}+\left(G_{y} f(x, y)\right)^{2}}
$$




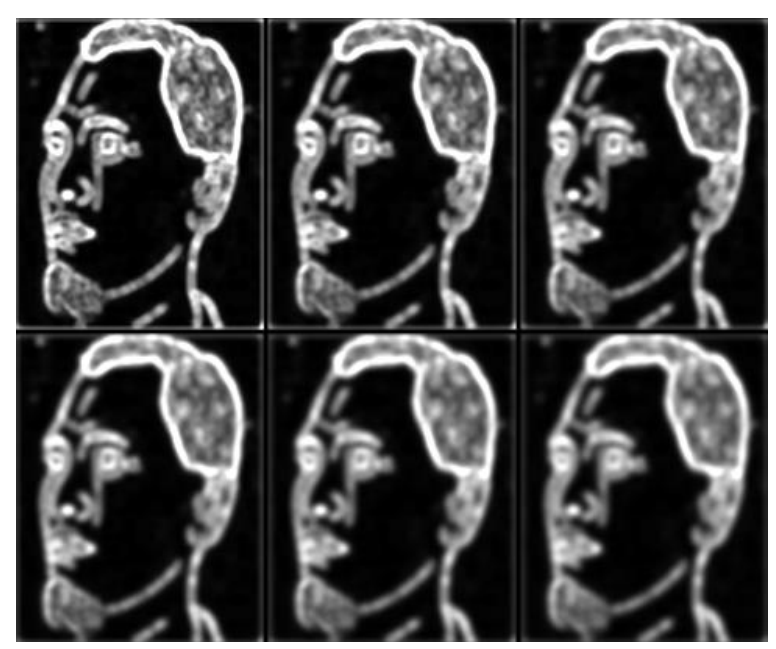

Figure 2 Image gradation result by using different number of iteration.

\subsection{The shapes Movement [6][7]}

In this research, shape movement is performed twice. On the first movement, the result of image gradation will be used to move shapes toward the corresponding features. The shape movement is based on improvement of the maximum gradient value. The landmarks move toward area which has the biggest maximum gradient value. The last, the results of the first movement will be used to the shape initialization. The shape movement will be controlled by using eight movement directions. Formula used to move landmarks toward the corresponding feature is the similarity transformation as written in the following equation:

$$
\left(t x_{i}^{\prime}, t y_{i}^{\prime}\right)=M\left(\theta, s_{x}, s_{y}\right)\left[t x_{i}, t y_{i}\right]+\left(t_{x}, t_{y}\right)
$$

\subsection{Improvement of the Line Gradient Method [7]}

On the previous research the line gradient was improved, but the direction of the movement cannot be controlled. On the previous research [7], the maximum line gradient value between the $i^{\text {th }}$ and the $(i+1)^{\text {th }}$ landmark can be calculated by using the following equation:

$$
\begin{aligned}
& \text { if }\left|y t_{i}-y t_{i+1}-\frac{1}{2}\right| y t_{i}+y t_{i+1}||>\left|x t_{i}-x t_{i+1}-\frac{1}{2}\right| x t_{i}+x t_{i+1}|| \text { then } \\
& \Delta x y=\left|y t_{i}-y t_{i+1}-\frac{1}{2}\right| y t_{i}+y t_{i+1}||
\end{aligned}
$$




$$
\begin{aligned}
& \text { if }\left|y t_{i}+y t_{i+1}+\frac{1}{2}\right| y t_{i}-y t_{i+1}||>\left|x t_{i}+x t_{i+1}+\frac{1}{2}\right| x t_{i}-x t_{i+1}|| \text { then } \\
& \Delta x y=\left|y t_{i}+y t_{i+1}+\frac{1}{2}\right| y t_{i}-y t_{i+1} \mid \\
& \text { if }\left|y t_{i}-y t_{i+1}-\frac{1}{2}\right| y t_{i}+y t_{i+1}|| \leq\left|x t_{i}-x t_{i+1}-\frac{1}{2}\right| x t_{i}+x t_{i+1}|| \text { then } \\
& \Delta x y=\left|x t_{i}-x t_{i+1}-\frac{1}{2}\right| x t_{i}+x t_{i+1}|| \\
& \text { if }\left|y t_{i}+y t_{i+1}+\frac{1}{2}\right| y t_{i}-y t_{i+1}|| \leq\left|x t_{i}+x t_{i+1}+\frac{1}{2}\right| x t_{i}-x t_{i+1}|| \text { then } \\
& \Delta x y=\left|x t_{i}+x t_{i+1}+\frac{1}{2}\right| x t_{i}-x t_{i+1}||
\end{aligned}
$$

To obtain the line gradient between the first and the last landmark can be calculated by using the following equation:

$$
\begin{aligned}
& \text { if }\left|y t_{n}-y t_{1}-\frac{1}{2}\right| y t_{n}+y t_{1}||>\left|x t_{n}-x t_{1}-\frac{1}{2}\right| x t_{n}+x t_{1}|| \text { then } \\
& \Delta x y=\left|y t_{n}-y t_{1}-\frac{1}{2}\right| y t_{n}+y t_{1}|| \\
& \text { if }\left|y t_{n}-y t_{1}-\frac{1}{2}\right| y t_{n}+y t_{1}|| \leq\left|x t_{n}-x t_{1}-\frac{1}{2}\right| x t_{n}+x t_{1}|| \text { then } \\
& \Delta x y=\left|x t_{n}-x t_{1}-\frac{1}{2}\right| x t_{n}+x t_{1}|| \\
& \text { if }\left|y t_{n}+y t_{1}+\frac{1}{2}\right| y t_{n}-y t_{1}||>\left|x t_{n}+x t_{1}+\frac{1}{2}\right| x t_{n}-x t_{1}|| \text { then } \\
& \Delta x y=\left|y t_{n}+y t_{1}+\frac{1}{2}\right| y t_{n}-y t_{1}||
\end{aligned}
$$




$$
\begin{aligned}
& \text { if }\left|y t_{n}+y t_{1}+\frac{1}{2}\right| y t_{n}-y t_{1}|| \leq\left|x t_{n}+x t_{1}+\frac{1}{2}\right| x t_{n}-x t_{1}|| \text { then } \\
& \Delta x y=\left|x t_{n}+x t_{1}+\frac{1}{2}\right| x t_{n}-x t_{1}||
\end{aligned}
$$

The results of Eq. (16) until (23) can be utilized to compute the gradient value along line as seen on the following equation:

$$
\begin{aligned}
& x t_{l}=x t_{i}+l / d *\left(x t_{i+1}-x t_{i}\right)+\sqrt{\left(x t_{i+1}+x t_{i}\right)} \\
& y t_{l}=y t_{i}+l / d *\left(y t_{i+1}-y t_{i}\right)+\sqrt{\left(y t_{i+1}+y t_{i}\right)}
\end{aligned}
$$

In this case, landmark between $\left(x t_{i}, y t_{i}\right)$ and $\left(x t_{i+1}, y t_{i+1}\right)$ is represented by using $d$. The maximum line gradient along line can be re-evaluate by using the following equation:

$$
\begin{aligned}
& \text { if } \Delta x y<d-1 \text {, then } L G(x t)=\bar{G}(x t) *(d-1) / \alpha(d-1+\beta) \\
& \text { else } L G(x t)=\bar{G}(x t) \\
& \text { if } \Delta x y<d-1 \text { then } L G(y t)=\bar{G}(y t)^{*}(d-1) / \alpha(d-1+\beta) \\
& \text { else } L G(y t)=\bar{G}(y t)
\end{aligned}
$$

In this case, $\bar{G}(x t)$ and $\bar{G}(y t)$ are average value of gradient along line of $x t$ and $y t$, respectively. Whereas $\alpha$ represents constant value between 0.1 until 1 , and $\beta=1 / \alpha$. The value of $\bar{G}(x t)$ and $\bar{G}(y t)$ can be calculated by using the following equation:

$$
\begin{aligned}
& \bar{G}(x t)=\frac{\sum_{i d=1}^{d} x t_{i d}}{d} \\
& \bar{G}(y t)=\frac{\sum_{i d=1}^{d} y t_{i d}}{d}
\end{aligned}
$$




\subsection{Integrating the Level Set Method Variation and Improvement of Maximum Line Gradient Method to Detect Multi Features as the First Movement [7]}

On the previous research [7], combining the results of modeling improvements according to Eq. (26) and (27) with level set variation method based on signed distance can be performed by using the following equations:

$$
\begin{gathered}
|\Phi(\overrightarrow{L G(x t)})|=d(\overrightarrow{L G(x t)}) \forall \overrightarrow{L G(x t)} . \\
\Phi=\left\{\begin{array}{l}
\text { less than } 0 \text { if } \overrightarrow{L G(x t)} \in \Omega^{-} \\
\text {greater than } 0 \text { if } \overrightarrow{L G(x t)} \in \Omega^{+} \\
\text {equal } 0 \text { if } \overrightarrow{L G(x t)} \in \partial \Omega
\end{array}\right.
\end{gathered}
$$

Surface line can be directly calculated by using the following equation:

$$
\left[N=\frac{\nabla \varphi}{|\nabla \varphi|}\right]
$$

\subsection{Enhancement of the Adaptive Shape Variants Average Values by Using Eight Movement Directions as the Second Movement}

Not all the landmarks of the first movement results occupy the exact feature points; therefore it is necessary to improve landmark movement. Novel approach to improve previous research based on the adaptive shape variation average values by using eight movement directions as second movement is proposed. If the number of landmarks for the $a$ th shape is $n_{a}$, then the number of landmark variations on each shape variation can be modeled by using the following equations:

$$
\begin{aligned}
& S \overline{\sigma x}_{1}=\left[\begin{array}{lll}
\overline{\sigma x}_{1} & \overline{\sigma x}_{2} & \ldots \ldots . . \bar{x}_{n_{1}}
\end{array}\right] \\
& S \overline{\mathscr{y}}_{1}=\left[\begin{array}{lll}
\overline{\mathscr{y}}_{1} & \overline{\sigma y}_{2} & \ldots . . . \overline{\sigma y}_{n_{1}}
\end{array}\right] \\
& S \bar{\sigma}_{2}=\left[\begin{array}{lll}
\bar{\sigma}_{n_{1}+1} & \bar{\sigma}_{n_{1}+2} & \ldots . . . \overline{\sigma x}_{n_{2}}
\end{array}\right] \\
& S \overline{\sigma y}_{2}=\left[\begin{array}{llll}
\overline{\sigma y}_{n_{1}+1} & \overline{\sigma y}_{n_{1}+2} & \ldots . . . \overline{\sigma y} \bar{n}_{2}
\end{array}\right]
\end{aligned}
$$

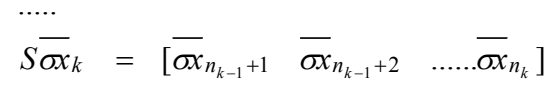

$$
\begin{aligned}
& S \bar{\sigma}_{k}=\left[\begin{array}{lll}
\overline{\sigma y}_{n_{k-1}+1} & \overline{\sigma y}_{n_{k-1}+2} & \ldots . . . \overline{\sigma y} \\
n_{k}
\end{array}\right]
\end{aligned}
$$


Based on Eq. (33) the shape variation average value can be calculated by using the following equations:

$$
\begin{aligned}
& {\overline{S \sigma x_{k}}}_{=}=\frac{\sum_{a=n_{k}-1}^{n_{k}} \overline{\sigma x}_{a}}{n_{k}-n_{k-1}+1} \\
& {\overline{S \sigma y_{k}}}_{k}=\frac{\sum_{a=n_{k}-1}^{n_{k}} \overline{\sigma y}_{a}}{n_{k}-n_{k-1}+1}
\end{aligned}
$$

To improve the previous research, we proposed the shape movement to 8 directions by using the following equation rule, which are upward, right, downward, left, up-right, down-right, down-left and up-left respectively as seen Figure 3.

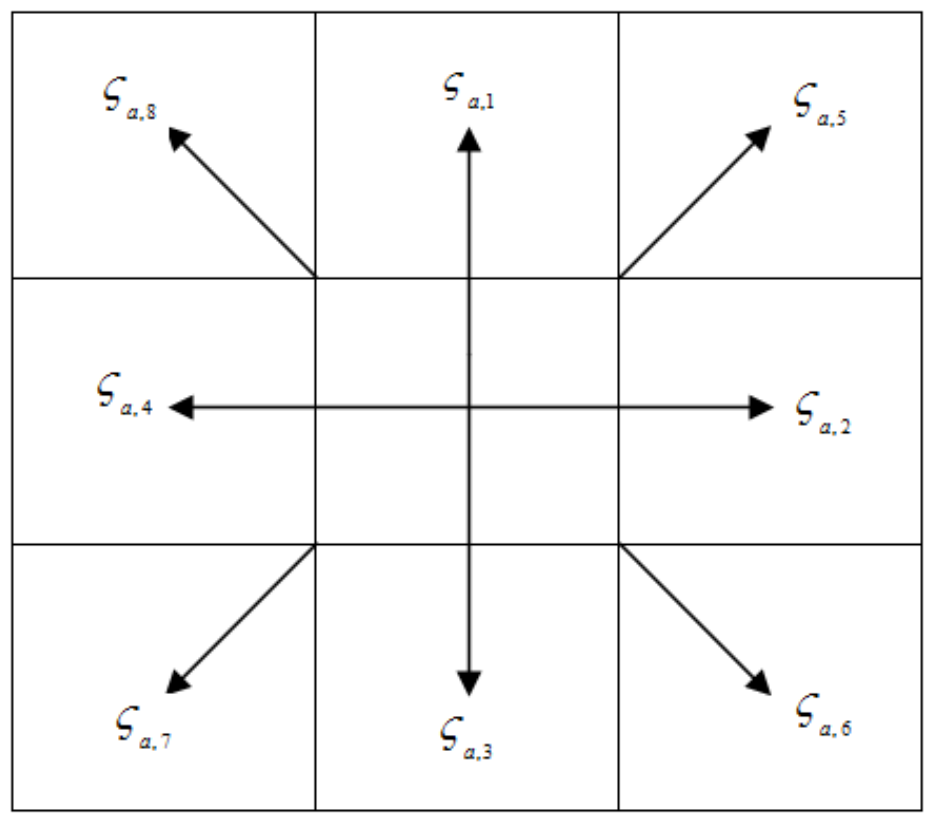

Figure 3 Description of landmark movement with 8 directions.

For upward direction, $p$ value used is 1 . 
If $\overline{S \sigma x}_{a 1}<\overline{S \sigma x}_{a}-\sqrt{\left(\overline{S \sigma x}_{a 1}+\overline{S \sigma x}_{a}\right)}$ and $\left|\overline{S \sigma x}_{a 1}-\overline{S \sigma x}_{a}-\sqrt{\left(\overline{S \sigma x}_{a 1}+\overline{S \sigma x}_{a}\right)}\right|<c M_{a, p}$ and $\left|\overline{S \sigma y}_{a 1}-\overline{S \sigma y}_{a}-\sqrt{\left(\overline{S \sigma x}_{a 1}+\overline{S \sigma x}_{a}\right)}\right|<r J$, then direction of shape movement can be expressed by using the following equation:

$$
\varsigma_{a, 1}=\overline{S \sigma x}_{a 1}-\overline{S \sigma x}_{a}-\sqrt{\left(\overline{S \sigma x}_{a 1}+\overline{S \sigma x}_{a}\right)}
$$

For the right direction, $p$ value used is 2 .

If $\overline{S \sigma x}_{a 1}>\overline{S \sigma x}_{a}-\sqrt{\left(\overline{S \sigma x}_{a 1}+\overline{S \sigma x}_{a}\right)}$ and $\left|\overline{S \sigma x}_{a 1}-\overline{S \sigma x}_{a}-\sqrt{\left(\overline{S \sigma x}_{a 1}+\overline{S \sigma x}_{a}\right)}\right|<c M_{a, p}$ and $\left|\overline{S \sigma y}_{a 1}-\overline{S \sigma y}_{a}-\sqrt{\left(\overline{S \sigma x}_{a 1}+\overline{S \sigma x}_{a}\right)}\right|<r J$, then direction of shape movement can be expressed by using the following equation:

$$
\varsigma_{a, 2}=\overline{S \sigma x}_{a 1}-\overline{S \sigma x}_{a}+\sqrt{\left(\overline{S \sigma x}_{a 1}+\overline{S \sigma x}_{a}\right)}
$$

For downward direction, $p$ value used is 3 .

If $\overline{S \sigma y}_{a 1}<\overline{S \sigma y}_{a}-\sqrt{\left(\overline{S \sigma y}_{a 1}+\overline{S \sigma y}_{a}\right)}$ and $\left|\overline{S \sigma y}_{a 1}-\overline{S \sigma y}_{a}-\sqrt{\left(\overline{S \sigma y}_{a 1}+\overline{S \sigma y}_{a}\right)}\right|<c M_{a, p}$ and $\left|\overline{S \sigma y}_{a 1}-\overline{S \sigma y}_{a}-\sqrt{\left(\overline{S \sigma y}_{a 1}+\overline{S \sigma y}_{a}\right)}\right|<r J$, then direction of shape movement can be expressed by using the following equation:

$$
\varsigma_{a, 3}=\overline{\operatorname{S\sigma y}}_{a 1}-\overline{\operatorname{Soy}}_{a}-\sqrt{\left(\overline{S \sigma y}_{a 1}+\overline{S \sigma y}_{a}\right)}
$$

For the left direction, $p$ value used is 4 .

If $\overline{S \sigma y}_{a 1}>\overline{S \sigma y}_{a}-\sqrt{\left(\overline{S \sigma y}_{a 1}+\overline{S \sigma y}_{a}\right)}$ and $\left|\overline{S \sigma y}_{a 1}-\overline{S \sigma y}_{a}-\sqrt{\left(\overline{S \sigma y}_{a 1}+\overline{S \sigma y}_{a}\right)}\right|<c M_{a, p}$ and $\left|\overline{S \sigma y}_{a 1}-\overline{S \sigma y}_{a}-\sqrt{\left(\overline{S \sigma y}_{a 1}+\overline{S \sigma y}_{a}\right)}\right|<r J$, then direction of shape movement can be expressed by using the following equation:

$$
\varsigma_{a, 4}=\overline{S \sigma y}_{a 1}-\overline{S \sigma y}_{a}+\sqrt{\left(\overline{S \sigma y}_{a 1}+\overline{S \sigma y}_{a}\right)}
$$

For the right-upper direction, $p$ value used is 5 . 
If $\overline{S \sigma x}_{a 1}<\overline{S \sigma x}_{a}+\sqrt{\left(\overline{S \sigma x}_{a 1}-\overline{S \sigma x}_{a}\right)}$ and $\left|\overline{S \sigma x}_{a 1}+\overline{S \sigma x}_{a}+\sqrt{\left(\overline{S \sigma x}_{a 1}-\overline{S \sigma x}_{a}\right)}\right|<c M_{a, p}$ and $\left|{\overline{S \sigma y_{a 1}}}_{1}+\overline{S \sigma y}_{a}+\sqrt{\left(\overline{S \sigma x}_{a 1}-\overline{S \sigma x}_{a}\right)}\right|<r J$, then direction of shape movement can be expressed by using the following equation:

$$
\varsigma_{a, 5}=\overline{S \sigma x}_{a 1}+\overline{S \sigma x}_{a}-\sqrt{\left(\overline{S \sigma x}_{a 1}-\overline{S \sigma x}_{a}\right)}
$$

For the right-bottom direction, $p$ value used is 6 .

If $\overline{S \sigma x}_{a 1}>\overline{S \sigma x}_{a}+\sqrt{\left(\overline{S \sigma x}_{a 1}-\overline{S \sigma x}_{a}\right)}$ and $\left|\overline{S \sigma x}_{a 1}+\overline{S \sigma x}_{a}+\sqrt{\left(\overline{S \sigma x}_{a 1}-\overline{S \sigma x}_{a}\right)}\right|<c M_{a, p}$ and $\left|{\overline{S \sigma y_{a 1}}}+\overline{S \sigma y}_{a}+\sqrt{\left(\overline{S \sigma x}_{a 1}-\overline{S \sigma x}_{a}\right)}\right|<r J$, then direction of shape movement can be expressed by using the following equation:

$$
\varsigma_{a, 6}=\overline{S \sigma x}_{a 1}+\overline{S \sigma x}_{a}-\sqrt{\left(\overline{S \sigma x}_{a 1}-\overline{S \sigma x}_{a}\right)}
$$

For the left-bottom direction, $p$ value used is 7.

If $\overline{S \sigma y}_{a 1}>\overline{S \sigma y}_{a}+\sqrt{\left(\overline{S \sigma y}_{a 1}-\overline{S \sigma y}_{a}\right)}$ and $\left|\overline{S \sigma y}_{a 1}+\overline{S \sigma y}_{a}+\sqrt{\left(\overline{S \sigma y}_{a 1}-\overline{S \sigma y}_{a}\right)}\right|<c M_{a, p}$ and $\left|\overline{S \sigma y}_{a 1}+\overline{S \sigma y}_{a}+\sqrt{\left(\overline{S \sigma y}_{a 1}-\overline{S \sigma y}_{a}\right)}\right|<r J$, then direction of shape movement can be expressed by using the following equation:

$$
\varsigma_{a, 7}=\overline{S \sigma y}_{a 1}+\overline{S \sigma y}_{a}-\sqrt{\left(\overline{S \sigma y}_{a 1}+\overline{S \sigma y}_{a}\right)}
$$

For the left-upper, $p$ value used is 8 .

If $\overline{S \sigma y}_{a 1}<\overline{S \sigma y}_{a}+\sqrt{\left(\overline{S \sigma y}_{a 1}-\overline{S \sigma y}_{a}\right)}$ and $\left|\overline{S \sigma y}_{a 1}+\overline{S \sigma y}_{a}+\sqrt{\left(\overline{S \sigma y}_{a 1}-\overline{S \sigma y}_{a}\right)}\right|<c M_{a, p}$ and $\left|\overline{S \sigma y}_{a 1}+\overline{S \sigma y}_{a}+\sqrt{\left(\overline{S \sigma y}_{a 1}-\overline{S \sigma y}_{a}\right)}\right|<r J$, then direction of shape movement can be expressed by using the following equation:

$$
\varsigma_{a, 8}=\overline{S \sigma y}_{a 1}+\overline{S \sigma y}_{a}+\sqrt{\left(\overline{S \sigma y}_{a 1}+\overline{S \sigma y}_{a}\right)}
$$


In this case $\zeta_{\mathrm{a}, \mathrm{p}}, p$ index of $\varsigma$ represents the direction, $p=1,2,3,4,5,6,7$ and 8 . For each $p$ value represents upward, right, downward, left, up-right, down-right, down-left and up-left respectively. Before Eq. (33) until (39) is applied, the value of $c M_{a, p}$ is defined by a great positive integer and replaced with $\varsigma_{a, p}$ from the previous process, in this case $r J$ is the integer value defined by the user.

\section{Experimental Results and Discussion}

In this research, we use 7 parameters, which are scaling $\left(s_{x}\right.$ and $\left.s_{y}\right)$, translation $\left(t_{x}\right.$ and $\left.t_{y}\right)$, rotation $(\theta)$, adjustment constant value of the shape initialization $(\eta)$, constant value is represented by using $\alpha, \beta=1 / \alpha$, range distance (integer value) is represented by using $r J$ and $c M$ represents cluster minimum distance. The parameter values used can be shown in Table 1.

Table 1 the parameter values used for our proposed method.

\begin{tabular}{ccc}
\hline Number & Symbol & Values \\
\hline 1 & $s_{x}$ and $s_{y}$ & 0.8 until 1.2 \\
2 & $t_{x}$ and $t_{y}$ & $-(+4)$ until $(+4)$ \\
3 & $\theta$ & $-15^{0}$ until $15^{0}$ \\
4 & $\eta$ & 0.15 \\
5 & $\alpha$ & 1.5 \\
6 & $r J$ & 10 \\
7 & $c M$ & 999 \\
\hline
\end{tabular}

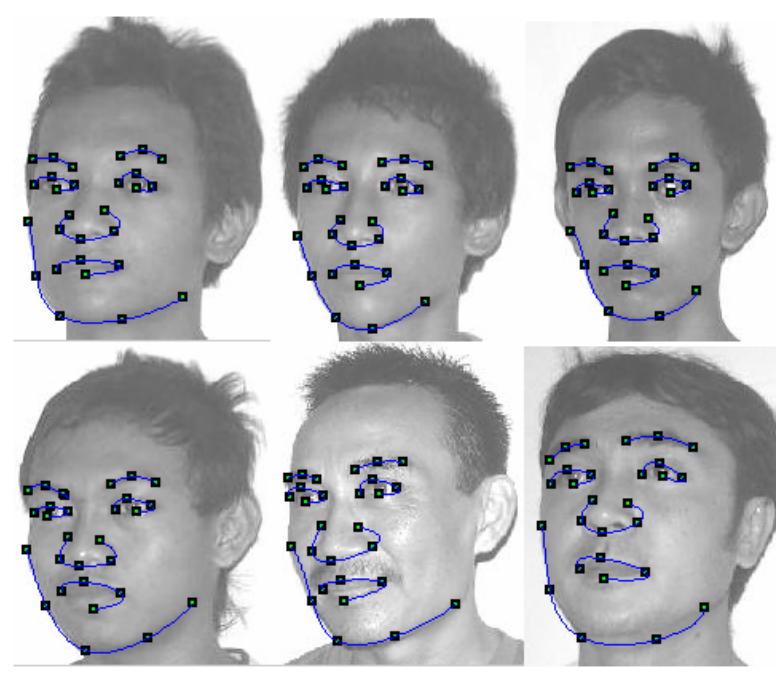

Figure 4 Training set of six different persons. 


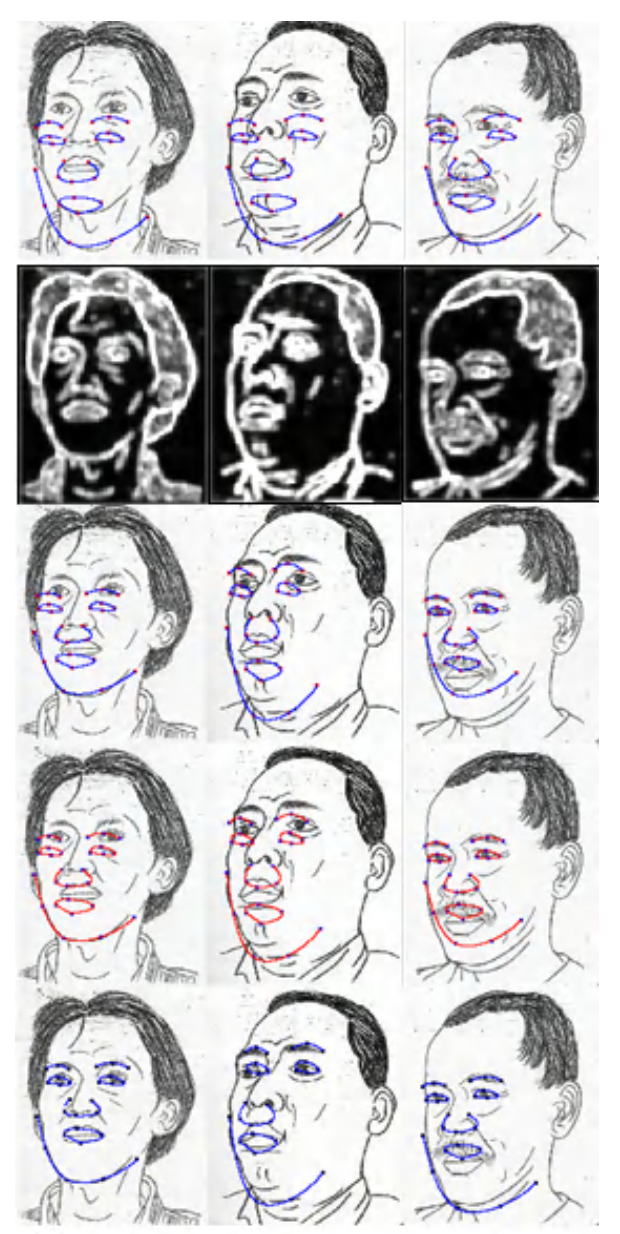

Figure 5 Part of our proposed method experimental results.

In order to test our proposed method, we use The Pentax camera to obtain 100 people photograph face image as training set, each person has limited tilt up to about 15 degrees. Photograph face images consist of 83 men and 17 women, while the expression of shooting time is by the normal expression. Facial image photograph capturing is done by using almost the same lighting from one person to each other. The distance between the camera and the person who photographed about $150 \mathrm{~cm}$. For each facial image photograph obtained is 2560x1920 pixels by photographer. This image is cropped on the face image part only. The cropping results is converted into the gray scale image and resized become $202 \times 162$ pixels. We employed 100 face sketches limited tilt up to about 15 degrees as testing set, these are resulted by sketcher. For face sketch image is digitized $1427 \times 918$ pixels and resized becomes $202 \times 162$ pixels. The larger image size processed, the longer time consumed. To anticipate it, it is 
necessary to change training and testing set becomes $202 \times 162$ pixels. We have put 28 landmarks of 7 shapes for all training set. Composite of the landmarks on each shape is considered as feature. The right and the left eyebrow consist of 3 landmarks respectively; the right and left eyes consist of 4 landmarks respectively; the nose consists of 5 landmarks; the lips consists of 4 landmarks and the face curvature consists of 5 landmarks as seen Figure 4. It is part of training set used in this research.

Small part of the experimental results can be seen in Figure 5 which contains 3 persons. The first row represents the landmark initialization on the testing set. The second row shows the results of creating image gradation. The third row represents the results of the improvement of landmark initialization by using Eqs. (5) and (6). The fourth row shows the results of Integrating the level set method variation and improvement of maximum line gradient method to detect multi features as the first movement [7], it is clear that shapes cannot move to the corresponding features. The last row represents the results of proposed method which is enhancement of the adaptive shape variants average values by using eight movement directions as the second movement. In the last two rows on the first person, there was improvement on the feature detection results, which are eyebrows, eyes, nose and lips. On the second person, there was improvement on the feature detection results, which are eyebrows and eyes. On the last person, there was improvement on the face curvature only. Inability to move the shape to the corresponding features was caused by the energy of non feature object greater than the corresponding features that will be detected.

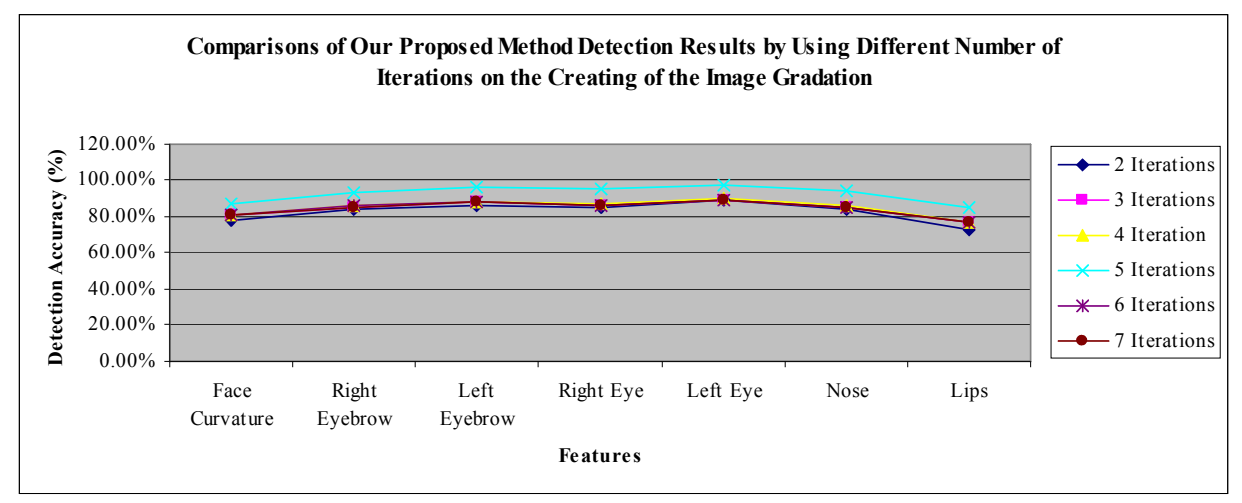

Figure 6 Comparisons of our proposed method detection results using different number of iterations on the creating of the image gradation. 
Table 2 The experimental results of face sketch multi feature detection using our proposed method.

\begin{tabular}{cccc}
\hline \multirow{2}{*}{ Number } & Features & \multicolumn{2}{c}{ Percentage } \\
\cline { 3 - 4 } & & $\begin{array}{c}\text { The First Movement } \\
{[7]}\end{array}$ & Proposed Method \\
\hline 1 & Face Curvature & $82.60 \%$ & $87.00 \%$ \\
2 & Right Eyebrow & $91.33 \%$ & $93.67 \%$ \\
3 & Left Eyebrow & $94.67 \%$ & $96.33 \%$ \\
4 & Right Eye & $93.00 \%$ & $95.25 \%$ \\
5 & Left Eye & $96.00 \%$ & $97.50 \%$ \\
6 & Nose & $91.20 \%$ & $94.40 \%$ \\
7 & Lips & $79.75 \%$ & $85.00 \%$ \\
\hline & Average & $89.79 \%$ & $92.74 \%$ \\
\hline
\end{tabular}

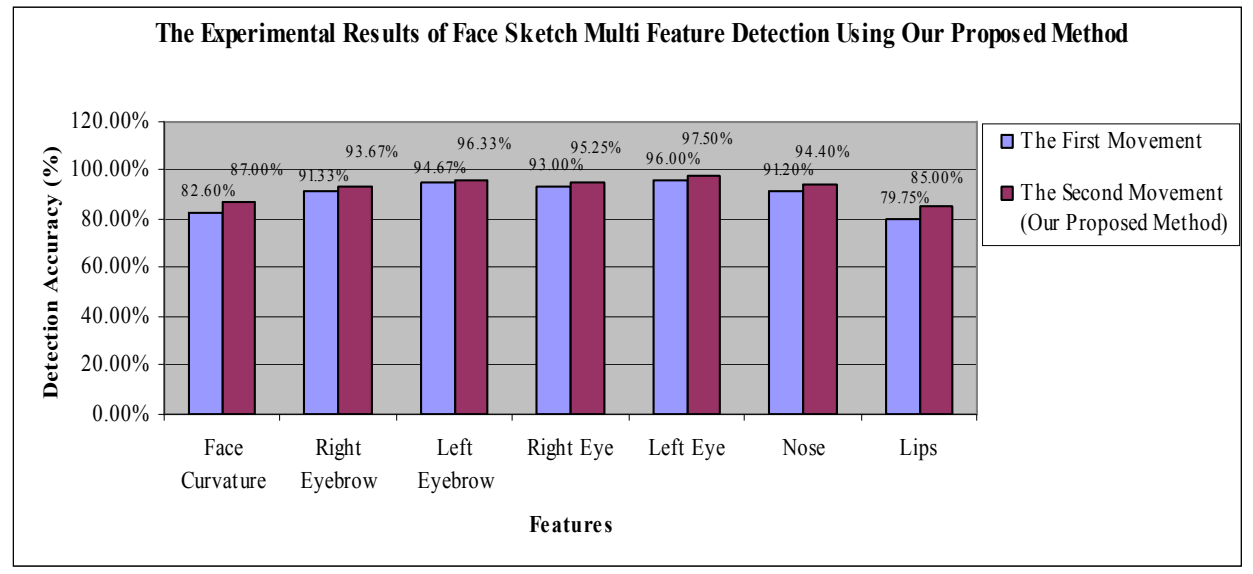

Figure 7 Experimental results of face sketch multi features detection using our proposed method.

Figure 6 shows the experimental results by using the different image gradation, optimal accuracy results can be achieved by using 5 iterations of image gradation. If number of iterations used less than 5, shapes movement cannot approach the corresponding features. If number of iterations used greater than 5 , shapes move toward incorrect area. The most appropriate iteration used is 5 as seen in Table 2 related to Figure 7. Table 2 and Figure 7 show that the detection percentage increases in the second movement compared to the first movement for all features. Our proposed method prove that, by the shape movement controlling using 8 eight directions can increase the result detection. The largest error occurred in the lips feature, which is $100 \%-85 \%=15 \%$. Whereas the smallest error occurred in the right eye feature, which is $100 \%-97.5 \%=2.5 \%$. Although the most major errors are found on the features of the lips, the feature 
experiences the greatest increase in detection accuracy, which is $85 \%$ $79.75 \%=5.25 \%$. While the features of the left eye, although the feature detection produces the greatest accuracy (97.5\%), but increase in detection accuracy of the second movement only $1.5 \%$.

\section{Conclusions and Future Work}

In this paper, it can be concluded that:

1. To move shape toward correspondence features, the ideal number of iterations used on gradation image was 5 . If the number of iterations used is less than 5, shapes movement cannot approach the corresponding features. If the number of iterations used is greater than 5, shapes move toward incorrect area.

2. The weakness of our proposed method is the inability to move shape towards the corresponding feature when shapes have to pass through objects that are not the dominant feature of the face. The simulation results has shown that the feature stops at objects rather than facial features when the shape is closer to the object and that object has a greater energy than the corresponding features.

If the adjustment of the value of translation, scale and rotation is not big enough, then the shape Initialization results will move away from features detected. For future work, it is necessary to improve shape initialization modeling. It can be conducted by detecting one feature position and followed by other features.

\section{Acknowledgment}

Author would like to thank to the Indonesian Directorate General of Higher Education (DIKTI), especially to DP2M department for its financial support under the Competition Grand 2010-2011.

\section{References}

[1] Muntasa, Arif, Hariadi, Mochamad \& Purnomo, Mauridhi Hery, Maximum Feature Value Selection of Nonlinear Function Based on Kernel Pca for Face Recognition, Proceeding of The 4th Conference on Information \& Communication Technology and Systems, Surabaya, Indonesia, pp. 397-402, 2008.

[2] Wright, John, Yang, Allen Y., Ganesh, Arvind, Sastry, S. Shankar \& Ma, Yi, Robust Face Recognition Via Sparse Representation, IEEE Transactions On Pattern Analysis And Machine Intelligence, 31(2), pp 210-227, 2009. 
[3] Sinha, P., Balas, B., Ostrovsky, Y. \& Russell, R., Face Recognition by Humans: Nineteen Results All Computer Vision Researchers Should Know About, Proc. IEEE, 94(11), pp. 1948-1962, 2006.

[4] Liu, C., Capitalize on Dimensionality Increasing Techniques for Improving Face Recognition Grand Challenge Performance, IEEE Trans. Pattern Analysis and Machine Intelligence, 28(5), pp. 725-737, 2006.

[5] Ahonen, T., Hadid, A. \& Pietikainen, M., Face Description with Local Binary Patterns: Application to Face Recognition, IEEE Trans. Pattern Analysis and Machine Intelligence, 28(12), pp. 2037-2041, 2006.

[6] Muntasa, Arif, Hariadi, Mochamad \& Purnomo, Mauridhi Hery, A New Formulation of Face Sketch Multiple Features Detection Using Pyramid Parameter Model and Simultaneously Landmark Movement, IJCSNS International Journal of Computer Science and Network Security, 9(9), pp 249-260, 2009.

[7] Muntasa, Arif, Integrating the Maximum Line Gradient Value Improvement and the Level Set Method Variation to Detect Face Sketch Multi-Features, International Journal of Digital Image Processing, 3(7), 2010.

[8] Hidaka, A., Nishida, K. \& Kurita, T., Face Tracking by Maximizing Classification Score of Face Detector Based on Rectangle Features, Proc. IEEE Int'l Conf. Computer Vision Systems, 2006.

[9] Liu, Xiaoming, Discriminative Face Alignment, IEEE Transactions on Pattern Analysis and Machine Intelligence, 31(11), pp. 1941-1953, 2009.

[10] Muntasa, Arif, A Novel Approach for Face Sketch Recognition Based on the First Derivative Negative and 2D-DCT with Overlapping Model, International Journal of Image Processing (IJIP), 4(4), pp. 368-376, 2010.

[11] Wang, Xiaogang \& Tang, Xiaoou, Face Photo-Sketch Synthesis And Recognition, IEEE Transactions On Pattern Analysis And Machine Intelligence, 31(11), pp. 1955-1967, 2010.

[12] Zhong. J., Gao, X. \& Tian, C., Face Sketch Synthesis Using a E-Hmm and Selective Ensemble, Proc. IEEE Int'l Conf. Acoustics, Speech, and Signal Processing, 2007.

[13] Tang, Xiaoou \& Wang, Xiaogang, Face Sketch Recognition, IEEE Transactions on Circuits and Systems for Video Technology, 14(1), pp. 50-57, 2004.

[14] Cristinacce, D. \& Cootes, T.F., Facial Feature Detection and Tracking with Automatic Template Selection, Proc. 7th IEEE International Conference on Automatic Face and Gesture Recognition, pp. 429-434, 2006.

[15] Cristinacce, D. \& Cootes, T.F., Feature Detection and Tracking with Constrained Local Models, Proc. British Machine Vision Conference, 3, pp.929-938, 2006. 
[16] Cristinacce, D. \& Cootes, T.F., Boosted Active Shape Models, Proc. British Machine Vision Conference, 2, pp.880-889, 2007.

[17] Bagherian, E., Rahmat, R.W. \& Udzir, N.I., Extract of Facial Feature Point, IJCSNS International Journal of Computer Science and Network Security, 9(1), pp. 49-53, 2009. 